\title{
Local Government Management and Performance: A Review of Evidence
}

\author{
Richard M. Walker \\ City University of Hong Kong \\ Rhys Andrews \\ Cardiff University
}




\section{introduction}

- Local governments = public service delivery

- Public face of the state $=$ street-level bureaucrats

- Many theories of public management tested in local government

- Much evidence on management and performance from local government settings

- Productivity of local government

- Efficiency - size

- Determinants of improvement following NPM 


\section{contributions}

1. A comprehensive assessment of what is currently known about the effects of management on the performance of local governments by integrating the theories and evidence from the empirical research that have been published during the past forty years in the leading public administration journals

2. A critical assessment of the methods and findings of the extant studies will help to identify a research agenda that builds on the strengths of the current evidence base while addressing areas that require further attention 


\section{DV: Performance, a contested concept?}

- Dimensions:

- Efficiency, Effectiveness, Equity, Responsiveness $=\mathrm{V}$

- Accountability, human rights, probity $=x$

- Stakeholders

- Internal (managers)

- External (citizens, users, businesses)

- Data sources

- Perceptual (survey)

- Archival (administrative records)

- Good or bad performance is: "in the eye of the beholder" (Andrews et al. 2006, 29) 


\section{theories}

- Economic (economies of size) versus Political (responsiveness to the citizenry)

- Public choice and NPM extension of competitive market structures

- Generic theories of organization

- Three broad perspectives

- Economic - scale economies of 1950/60s and competitive market structures

- Contingency - fit: INLOGOV 1960/70s

- Resource-based views - capacity 1970s onwards and USA 


\section{local government management approaches}

\begin{tabular}{|c|c|c|c|c|}
\hline Theory & Key concepts & $\begin{array}{c}\text { O'Toole } \\
\text { and } \\
\text { Meier }\end{array}$ & $\begin{array}{l}\text { Anticipated } \\
\text { relationship } \\
\text { with } \\
\text { performance }\end{array}$ & $\begin{array}{l}\text { Included in } \\
\text { the meta- } \\
\text { analysis }\end{array}$ \\
\hline \multirow[t]{5}{*}{$\begin{array}{l}\text { Economic } \\
\text { theory }\end{array}$} & Organization size & $\begin{array}{c}\text { M1, } \\
\text { M3/M4 }\end{array}$ & + & $\checkmark$ \\
\hline & Contracting out & M3/M4 & - or + & \\
\hline & Competition & M3/M4 & - or + & \\
\hline & Collaboration & M3/M4 & + & \\
\hline & Coproduction & M3/M4 & + & \\
\hline \multirow{5}{*}{$\begin{array}{l}\text { Contingency } \\
\text { theory }\end{array}$} & Administrative & M1 & - & \\
\hline & intensity & M1 & - & \\
\hline & Centralization & M1 & + & \\
\hline & Strategy content & M3/M4 & + & $\checkmark$ \\
\hline & Planning & M1 & + & $\checkmark$ \\
\hline \multirow{7}{*}{$\begin{array}{l}\text { Resource- } \\
\text { based theories }\end{array}$} & Management systems & M1 & + & \\
\hline & Staff quality & M1 & + & $\checkmark$ \\
\hline & Personnel stability & M1 & + & $\checkmark$ \\
\hline & Leadership & M1 & + & \\
\hline & HRM practices & M1 & + & \\
\hline & $\begin{array}{l}\text { Representative } \\
\text { bureaucracy }\end{array}$ & M3/M4 & + & $\checkmark$ \\
\hline & Networking & M3/M4 & + & $\checkmark$ \\
\hline
\end{tabular}




\section{Methods}

- SSCI Web of Science search 1970-2012

- 490 studies of which 86 met search criteria

- DV = performance, IV = management, organization, empirical

- But ... not many studies published correlation coefficients so use coefficients from regression: 999 tests

- Support score method implemented 


\section{Support score}

- The percentage of statistical tests that support the hypothesis that management positively or negatively influences performance

- Results have to be:

1. In predicted direction 2. statistically significant

- Support score calculated in two ways

1. Equally by study, regardless of whether it contains 1 or 300 tests.

2. Each study can be weighted (multiplied) by the number of tests in that study, with equal weight attached to each test rather than to each study

- Support for hypotheses

- Strong if both support scores $>50 \%$

- Moderate if one support score is $>50 \%$

- Not supported when both scores fall $<50 \%$ 


\section{illustration of findings strategy content}

\begin{tabular}{|c|c|c|c|c|c|c|c|c|c|c|c|}
\hline \multirow[b]{2}{*}{ Study } & \multirow[b]{2}{*}{ Sample } & \multirow[b]{2}{*}{ Country } & \multirow{2}{*}{$\begin{array}{l}\text { Purpo } \\
\text { se }\end{array}$} & \multicolumn{2}{|c|}{ Management } & \multicolumn{2}{|c|}{ Performance } & \multirow{2}{*}{$\begin{array}{l}\text { No. } \\
\text { tests }\end{array}$} & \multirow[b]{2}{*}{+} & \multirow[b]{2}{*}{-} & \multirow[b]{2}{*}{ ns } \\
\hline & & & & Strategy & Measure & Dimension & Measure & & & & \\
\hline Folz \& Hazlett (1991) & 89 & USA & SP & Technical innovation & Archival & Eft & Archival & 1 & 100 & 0 & 0 \\
\hline Folz (2004) & 556 & USA & SP & Technical innovation & Archival & Eft & Archival & 1 & 100 & 0 & 0 \\
\hline Andrews et al. (2005) & 150 & UK & MP & $\begin{array}{l}\text { Defending, prospecting, } \\
\text { reacting }\end{array}$ & Perceptual & In, US & Archival & 9 & 67 & 0 & 33 \\
\hline $\begin{array}{l}\text { Andrews, Boyne \& } \\
\text { Walker (2006) }\end{array}$ & 119 & UK & MP & $\begin{array}{l}\text { Defending, prospecting, } \\
\text { reacting, change market, } \\
\text { change service, seek } \\
\text { revenues }\end{array}$ & Perceptual & In & Both & 3 & 67 & 0 & 33 \\
\hline Meier et al. (2007) & 3041 & USA & SP & $\begin{array}{l}\text { Defending, prospecting, } \\
\text { reacting }\end{array}$ & Perceptual & Eft, Eq, Q & Archival & 27 & 22 & 15 & 63 \\
\hline Andrews et al. (2008) & 51 & UK & MP & $\begin{array}{l}\text { Defending, prospecting, } \\
\text { reacting }\end{array}$ & Perceptual & Eft & Archival & 6 & 67 & 33 & 0 \\
\hline Andrews et al. (2009) & 47 & UK & MP & $\begin{array}{l}\text { Defending, prospecting, } \\
\text { reacting }\end{array}$ & Perceptual & Eft & Both & 3 & 100 & 0 & 0 \\
\hline Meier et al. (2010) & 3041 & USA & SP & $\begin{array}{l}\text { Defending, prospecting, } \\
\text { reacting }\end{array}$ & Perceptual & Eft & Archival & 6 & 33 & 17 & 50 \\
\hline $\begin{array}{l}\text { Walker, Andrews et al. } \\
\text { (2010) }\end{array}$ & 101 & UK & MP & $\begin{array}{l}\text { Defending, prospecting, } \\
\text { reacting }\end{array}$ & Perceptual & In & Both & 12 & 50 & 0 & 50 \\
\hline Walker et al. (2011) & 136 & UK & MP & Management innovation & Perceptual & In & Both & 4 & 0 & 0 & 100 \\
\hline Andrews et al. (2012) & 178 & UK & MP & $\begin{array}{l}\text { Defending, prospecting, } \\
\text { reacting }\end{array}$ & Perceptual & In & Both & 6 & 87 & 0 & 17 \\
\hline $\begin{array}{l}\text { Owens \& Kukla- } \\
\text { Acevedo (2012) }\end{array}$ & 2490 & USA & SP & Prospecting & Perceptual & Eft & Archival & 3 & 100 & 0 & 0 \\
\hline & & & & & & $\begin{array}{l}\text { Number o } \\
\text { Number o } \\
\text { Unweight } \\
\text { Weighted }\end{array}$ & $\begin{array}{l}\text { studies } \\
\text { tests } \\
\text { d }\end{array}$ & $\begin{array}{l}12 \\
75\end{array}$ & $\begin{array}{l}66 \\
47\end{array}$ & $\begin{array}{r}7 \\
11\end{array}$ & $\begin{array}{l}28 \\
43\end{array}$ \\
\hline $\begin{array}{l}\text { Key } \\
\text { Purpose: MP = multi-pu } \\
\text { Performance dimension: } \\
\text { Satisfaction. }\end{array}$ & $\begin{array}{l}\mathrm{e}, \mathrm{SP}=\mathrm{s} \\
=\text { Cost } \mathrm{E}\end{array}$ & $\begin{array}{l}\text { igle purpos } \\
\text { fectiveness }\end{array}$ & $\mathrm{Eft}=\mathrm{E}$ & ectiveness, Efy = Efficie & $\mathrm{Eq}=\mathrm{Equi}$ & Indo & uality, Q & 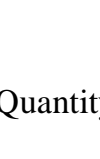 & $\mathrm{S}=$ & & \\
\hline
\end{tabular}




\section{main findings}

\begin{tabular}{l|lllllll} 
& \multicolumn{3}{|c}{ No. of } & \multicolumn{1}{c}{ Positive } & Negative \\
& Studies & Tests & U & W & U & W \\
\hline Organizational size & $\mathbf{2 0}$ & $\mathbf{5 8}$ & 34 & 26 & 10 & 3 \\
Strategy content & $\mathbf{1 2}$ & $\mathbf{7 5}$ & $\mathbf{6 6}$ & $\mathbf{4 7}$ & 7 & 11 \\
Planning & $\mathbf{1 4}$ & $\mathbf{1 3 8}$ & $\underline{\mathbf{6 2}}$ & $\underline{\mathbf{5 6}}$ & 2 & 3 \\
Staff quality & $\mathbf{1 4}$ & $\mathbf{8 1}$ & $\underline{\mathbf{7 3}}$ & $\underline{\mathbf{7 7}}$ & 3 & 5 \\
Personnel stability & $\mathbf{1 4}$ & $\mathbf{8 6}$ & $\underline{\mathbf{5 3}}$ & $\underline{\mathbf{5 2}}$ & 16 & 15 \\
Rep' bureaucracy & $\mathbf{1 4}$ & $\mathbf{1 2 9}$ & $\mathbf{6 1}$ & $\mathbf{4 3}$ & 13 & 9 \\
Managerial networking & $\mathbf{1 5}$ & $\mathbf{1 3 3}$ & $\mathbf{6 0}$ & $\mathbf{3 7}$ & $\mathbf{7}$ & 20
\end{tabular}




\section{sub-analysis}

- Single versus multiple forms of government

- Single $=53 / 43 \%$ (55 studies, 644 tests) (USA)

- Multiple purpose $=43 / 31 \%(31,355)$ (UK)

- Organizational echelons

- Representative bureaucracy: 18/20\% upper level and $67 / 60 \%$ front-line $(8,49)$

- Personnel stability: 54/56\% upper level, 59/48\% frontline $(8,34)$

- Archival versus perceptual performance

- Perceptions right: $49 / 43 \%(38,457)$

- Archival: 49/49\% (54, 457) 


\section{sub-analysis II: dimensions of performance:}

\begin{tabular}{|c|c|c|c|c|c|c|c|c|c|c|c|c|}
\hline \multirow[b]{2}{*}{ Management } & \multicolumn{3}{|c|}{ Efficiency } & \multicolumn{3}{|c|}{ Effectiveness } & \multicolumn{3}{|c|}{ Equity } & \multicolumn{3}{|c|}{ Index } \\
\hline & $S$ & $\mathrm{~T}$ & + & S & $\mathrm{T}$ & + & $S$ & $\mathrm{~T}$ & + & $S$ & $\mathrm{~T}$ & + \\
\hline Size & 7 & 20 & $50(40)$ & 5 & 16 & $4(6)$ & & & $X$ & 5 & 10 & $20(10)$ \\
\hline Strategy & & & X & 7 & 32 & $75(50)$ & & & $X$ & 5 & 22 & $60(59)$ \\
\hline Planning & & & $X$ & 5 & 56 & $80(82)$ & & & $X$ & 8 & 23 & $53(52)$ \\
\hline Staff quality & & & X & 13 & 46 & $73(78)$ & 6 & 27 & $90(93)$ & & & X \\
\hline Stability & & & X & 12 & 57 & $47(44)$ & 4 & 19 & $50(84)$ & & & $X$ \\
\hline Representative Bureaucracy & & & $X$ & 10 & 83 & $48(42)$ & 7 & 15 & $71(60)$ & & & $X$ \\
\hline Networking & & & $X$ & 14 & 58 & $73(60)$ & 4 & 58 & $29(31)$ & & & $X$ \\
\hline \multicolumn{13}{|l|}{ Key: } \\
\hline \multicolumn{13}{|c|}{ Weighted support scores in parenthesis } \\
\hline \multicolumn{13}{|c|}{$S=$ number of studies, $\mathrm{T}=$ number of tests, $+=$ positive support score } \\
\hline$X=$ no test & & & & & & & & & & & & \\
\hline
\end{tabular}




\section{summary of findings}

- Main findings

- Strong positive performance effects from staff quality, personnel stability, and planning

- Moderate support for the benefits of networking, representative bureaucracy and strategy content

- Weak in relation to organizational size

- Sub-analysis

- Single versus multipurpose: little difference

- Levels in the hierarchy matter

- Management approach has varying performance impact 


\section{discussion}

- Dataset dominated by studies from:

- UK: contingency theory (content and planning)

- USA: resource-based capacity views

- Full range of concept underlying theories that explain management and performance have not been tested

- Topics with limited:

- administrative intensity, collaboration/partnership, competition and leadership

- or no attention

- inter-organizational relations, decentralization of decision-making and bureaucratic autonomy 


\section{limitations}

- $\mathrm{SSCl}$ search

- PA based search: political science, urban studies, policy field journals ...

- Limited attention to research design

- Measurement

- Model specification

- Single versus multiple informants

- A plea for help:

- Publish correlation coefficients - can undertake more traditional approaches to meta analysis 